\title{
Muscle fatigue
}

\author{
R. H. T. EDWARDS \\ B.Sc., Ph.D., M.B., M.R.C.P.
}

Royal Postgraduate Medical School, Hammersmith Hospital, Du Cane Road, London

\begin{abstract}
Summary
Muscle fatigue is a common symptom but there are no universally accepted methods for quantitating the function of voluntary muscle. This paper describes three main methods of assessment: simple clinical tests of muscle function; thermal probe measurements of metabolic heat production during muscular contraction; needle biopsy studies of muscle structure and chemistry. These methods, though at a relatively early stage of development, have given promising results which suggest that they could be useful in assessing possible new forms of treatment in patients with neuromuscular disorders.
\end{abstract}

A Skeletal muscle is said to be fatigued when it fails to sustain the required force. The mechanisms controlling the force of contraction in man involve several interrelated physiological and psychophysical feedback control mechanisms (Fig. 1). The system of weakness and fatigue would seem likely in some way to stem from disturbances in these complex mechanisms. The physiology of fatigue has been studied at various levels of organization in animal muscle preparations (reviewed in Simonson, 1971) but there have been few advances which would allow a systematic investigation of human weakness and fatigue. Electromyography and allied electrophysiological techniques play an increasingly important role in the clinical diagnosis of neuromuscular disorders (Desmedt, 1973) but still the evaluation is to a large extent qualitative since quantitative EMG techniques, though potentially valuable, are still in their infancy.

The assessment of weakness and fatigue by the usual clinical examination of the neuromuscular system is difficult unless changes are gross. The Medical Research Council grading scale for classifying muscular contraction has been widely used since its introduction during the Second World War (Medical Research Council, 1943). This scale, though valuable for describing large changes, does not give a sufficiently objective assessment to allow precise

Based on a British Postgraduate Medical Federation 'Scientific Basis of Medicine' Lecture, 1974.

Correspondence: Dr R. H. T. Edwards, Royal Postgraduate Medical School, Hammersmith Hospital, Du Cane Road, London W12 0HS.

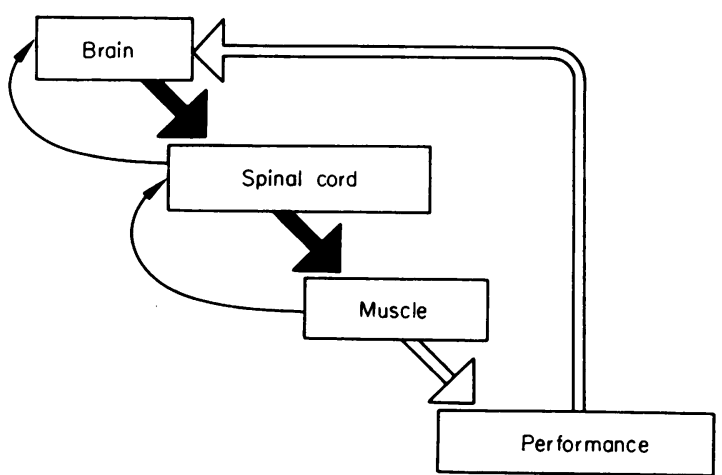

Fig. 1. Simple diagram of physiological and psychophysical feedback mechanisms controlling muscular contraction.

comparisons to be made on separate occasions by the same observer or by different observers. A simple quantitative test, which might be included in the clinical examination, is to time how long the patient when lying supine can hold one leg at $45^{\circ}$ above the couch. Normal subjects vary widely in the time they can do this but the lower limits found in 150 men and 150 women by Fessel, Taylor and Johnson (1970) were $60 \mathrm{sec}$ for the men and $30 \mathrm{sec}$ for the women. A similar test, studied by the same authors, is to time how long the head can be held off the couch, when the subject is lying supine. Lower normal limits found in the same populations were $90 \mathrm{sec}$ for the men and $30 \mathrm{sec}$ for the women.

Voluntary muscle power can now be quantitated in the course of the usual examination of the neuromuscular system with a new hand-held clinical dynamometer (Edwards and McDonnell, 1974). This instrument ('The Hammersmith Myometer') fits into the palm of the examiner's hand and registers the maximum force exerted on the patient's limb when a resisted movement is overcome. Clearly the force recorded depends on the mechanics of the muscle and lever systems operating. The myometer has to be applied to specified anatomical sites for measurements to be consistent. This instrument is potentially as useful an addition to the physicians' examination equipment as the sphygmomanometer and peak flow meter. Simple quantitative tests of 
muscle function are likely to become more important in the future since there is increasing emphasis on physiotherapeutic methods in rehabilitation and the promise of effective treatment of a number of locomotor disorders as a result of recent advances in neuropharmacology.

Such simple clinical tests can never elucidate the cause of fatigue. Moreover the measurement of voluntary contractions depends on the co-operation of the patient. The new approaches that I havesought to develop with my colleagues Dr D. K. Hill, F.R.S., Dr D. A. Jones and Mrs Caroline Maunder at the Royal Postgraduate Medical School are designed to overcome these problems and to break into the complex of interrelated servo-controls by establishing objective measurements of the metabolic activity of the contracting muscle. Fortunately fatigue is a symptom (unlike angina) which can be experienced under experimental conditions by healthy individuals (e.g. after muscular exercise). It has, therefore, been possible to develop the approach by studies in healthy volunteers before seeking possible application for the study of patients with myopathies.

First a suitable muscle must be chosen for the experimental studies. No muscle is ideal for this purpose but the quadriceps has the advantages that it is an important muscle for physical activities in everyday life, and it is affected to a greater or lesser extent in many myopathies. Further advantages are that the muscle (the vastus lateralis fraction of the quadriceps) is large enough and free of important blood vessels and nerves to allow probes to be inserted and for small samples of muscle to be safely taken by a needle biopsy technique (Bergström, 1962).

In normal life a muscle may shorten, lengthen or may remain at constant length during contraction. The first two forms of contraction are involved in rhythmic ('dynamic') exercise, such as walking or cycling whereas the last ('isometric') form is important in gripping or holding tasks. An isometric contraction of the quadriceps is studied with the subject seated in an adjustable chair. The pelvic girdle is stabilized with a seat belt and the force of contraction measured with a strain gauge attached to the ankle when the knee is flexed to a right angle (Tornvall, 1963). Intramuscular pressure rises and occludes the muscle circulation at forces greater than $20 \%$ of a maximum voluntary contraction (Barcroft and Millen, 1939; Edwards, Hill and McDonnell, 1972). This means that the energy for sustaining a long submaximal contraction must be almost entirely supplied from anaerobic sources (Table 1). Since no external work is done in this type of contraction, all the chemically stored energy which is exchanged during muscular activity has to appear as heat, which in the absence of blood flow is largely retained in the muscle. The quadriceps muscle thus behaves as a closed system' when it contracts isometrically and this provides a valuable experimental model for studying muscular contraction in man. Force is measured with a strain gauge, metabolic heat production is measured with a thermal probe (Edwards, McDonnell and Hill, 1974b) and changes in muscle metabolism are followed by chemical analysis of repeated needle biopsy samples (Hultman, 1967; Karlsson, 1971; Edwards et al., 1972a; Ahlborg et al., 1972).

It is well known that the time an isometric contraction can be sustained depends on the force exerted. From a large number of observations in several muscles, Rohmert (1960) defined a curved relation between endurance time and force (Fig. 2). Our results generally agree with Rohmert's, however, some of our athletes had a greater capacity to sustain contractions than our sedentary subjects, possibly because of superior motivation or because of metabolic adaptations in their muscles (Gollnick et al., 1972). The subject's full co-operation is needed in establishing the correct value for the force of a maximal voluntary contraction (MVC), the standard by which all submaximal force measurements are compared. In well motivated normal subjects the MVC force is proportional to the body weight (Fig. 3). (Body weight was chosen as a standard not only because muscular strength obviously varies with body size but because the quadriceps muscle plays an important role in supporting a large part of the body weight in everyday life activities.) In patients there is likely to be uncertainty as to the validity of MVC measurements and whether or not contractions have been

TABLE 1. Energy sources for muscular activity

Short term (anaerobic) sources

1. Adenosine triphosphate (ATP) $\longrightarrow$ adenosine diphosphate (ADP) + inorganic phosphate $\left(\mathbf{P}_{i}\right)+$ energy

2. Phosphorylcreatine + ADP $\rightleftharpoons$ creatine + ATP

3. Glycogen/glucose $+\mathbf{P}_{i}+\mathrm{ADP} \rightleftharpoons$ lactate + ATP

Long term (aerobic) sources

1. Glycogen/glucose $+\mathrm{ADP}+\mathrm{P}_{i}+\mathrm{O}_{2} \longrightarrow \mathrm{H}_{2} \mathrm{O}+\mathrm{CO}_{2}+\mathrm{ATP}$

2. Free fatty acids $+\mathrm{ADP}+\mathrm{P}_{i}+\mathrm{O}_{2} \rightarrow \mathrm{H}_{2} \mathrm{O}+\mathrm{CO}_{2}+\mathrm{ATP}$ 


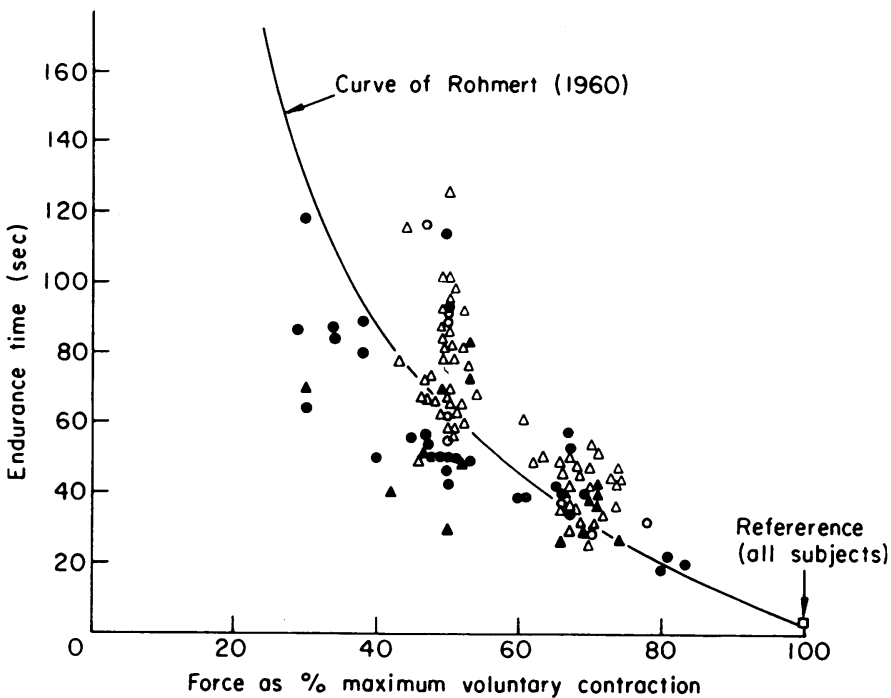

FIG. 2. Relationship between force of contraction and endurance time for isometric contractions of the quadriceps muscle, sustained to fatigue. Symbols indicate results in individual subjects studied at Hammersmith. The curve, redrawn from Rohmert (1960) is based on measurements in thirteen different muscle groups, including the quadriceps. Sedentary males, $(n=10)$; female subjects, $\Delta(n=5)$; male athletes, $O(n=5)$; female athletes, $\Delta(n=11)$.

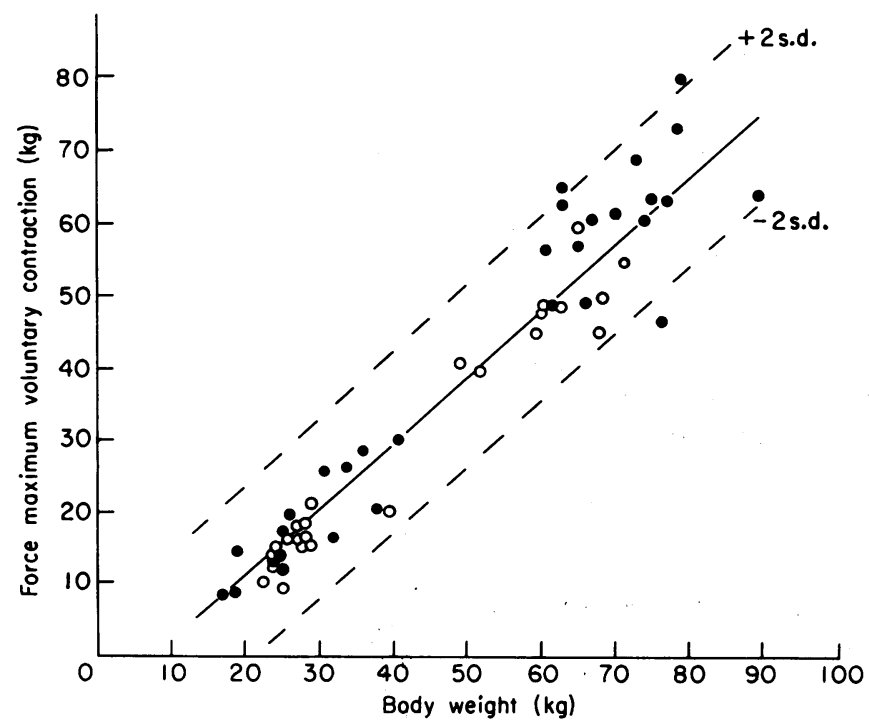

Fig. 3. The force of a maximum voluntary contraction of the quadriceps muscle. Results in children and adults related to body weight (obese subjects excluded). Normal subjects aged 5-38 $(\mathrm{n}=53)$ : males, ; females, O. $\mathrm{y}=0.91 \mathrm{x}-6.9, \mathrm{r}=0.952, P<0.001$. 


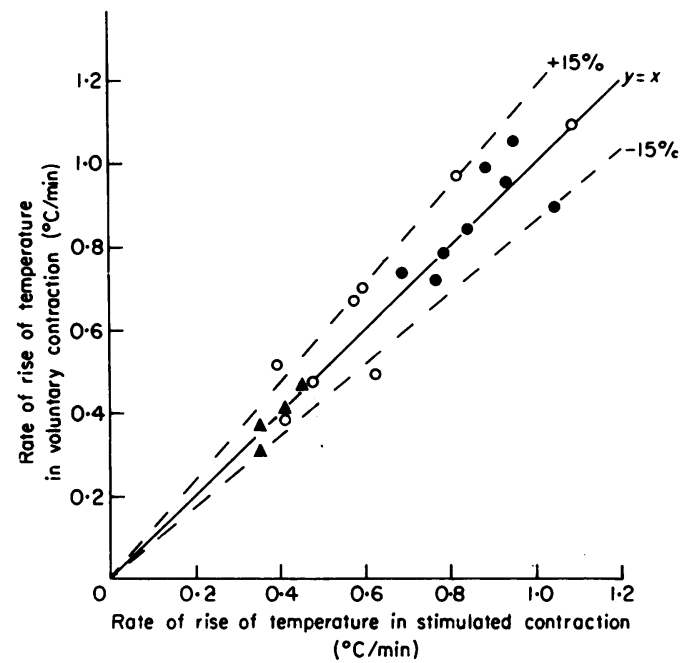

FIG. 4. Thermal probe measurements: rate of rise of temperature in maximum voluntary contractions compared with those in electrically stimulated contractions. Note that muscle can be maximally activated in a voluntary effort. Normal subjects: fresh muscles, $\bigcirc$; fatigued muscles, $\Delta$. Patients: fresh muscle, $O$.

properly sustained to 'fatigue' or stopped prematurely. The finding of a normal MVC force can be reassuring if the force is consistently low but the question is raised whether the patient would not or could not make a stronger contraction. These possibilities can be investigated further by artificially stimulating the muscle. The extent to which the muscle itself is working normally can then be determined by measuring the metabolic heat production with a thermal probe (Edwards et al., 1974b) and comparing the values obtained with those found during maximum voluntary contractions (Fig. 4). For this purpose a fraction $(20-40 \%)$ of the muscle is stimulated electrically via two skin electrodes placed proximally and distally on the anterior aspect of the thigh. This technique has been adopted since it is less painful than the arguably more reliable method of supramaximal stimulation of the femoral nerve. Consistent results have been found and show that the part of the quadriceps muscle into which the thermal probe is inserted can normally be maximally activated by a voluntary effort not only when the muscle is fresh but also when fatigued (Fig. 4). The patients with metabolic myopathies studied were also able to activate their muscles maximally in a voluntary effort although in several individuals the absolute values obtained in both types of contraction were lower than normal. Failure to achieve the same rate of temperature rise in a voluntary effort as with electrical stimulation suggests some disorder in the nervous control of muscular contraction rather than in the muscle itself. This could be at any level in the nervous system and other evidence is needed to decide whether the cause is psychological or the result of an organic lesion. If the rate of temperature rise is similar in both types of contraction but low, as when normal muscle is fatigued, there may' be a disorder of neuromuscular transmission, muscle metabolism or excitation-contraction coupling. The last two possibilities can be distinguished by measuring the contraction forces elicited with a range of stimulation frequencies (Edwards et al., 1974a) and by needle biopsy studies of muscle metabolism (see below). Failure of neuromuscular transmission can be recognized by examination of the force records (or muscle action potentials) during repetitive nerve stimulation (Desmedt, 1973) and by the Edrophonium test.

Thermal probes can now be made in the form of fine needles or thermocouple wires which can be inserted into almost any muscle. Myothermal techniques, though at present at a relatively early stage of development, may in the future provide useful information which is complementary to that obtainable by current electromyographic techniques.

Objective information about fatigue in sustained isometric contractions can be obtained by studying the changes in muscle chemistry during contraction. This requires chemical analysis of repeated needle biopsy samples. Accounts of the needle biopsy technique, its history and use in diagnosis and research have been published elsewhere (Edwards, 1971; Edwards et al., 1973) and practical details have been illustrated in a colour film with sound commentary (Edwards, 1973).

The immediate energy source for muscular activity is adenosine triphosphate (ATP) (Table 1). This is resynthesized continuously by breakdown of phosphorylcreatine, by anaerobic glycolysis and by oxidation of blood-borne glucose and free fatty acids. Occlusion of the circulation during an isometric contraction means that the oxidative energy contribution is limited to that resulting from the small amount of oxygen stored with myoglobin. This contribution was eliminated in our experimental study of anaerobic energy supply mechanisms during muscular contraction by inflating a thigh cuff to 200 $\mathrm{mmHg} 3 \mathrm{~min}$ before the start of the contraction. Needle biopsies were obtained immediately before and at the end of isometric contractions held at a force of $50 \% \mathrm{MVC}$, and sustained to fatigue. The patients studied presented at Medical Clinics complaining of muscle weakness and fatigue but histological, histochemical and electronmicroscopical examination of needle biopsy samples (Edwards et al., 1973) revealed no abnormalities. Muscle contents of ATP, phosphorylcreatine and lactate were the same in the patients as in the normal subjects both 
at rest and at fatigue (Fig. 5). This provides objective evidence that the patients were capable of driving their muscles to the same extent as the normal subjects and that energy supply processes were working normally. The complaint of 'fatigue' in these patients does not, therefore, appear to be due to abnormal energy supply during contraction.

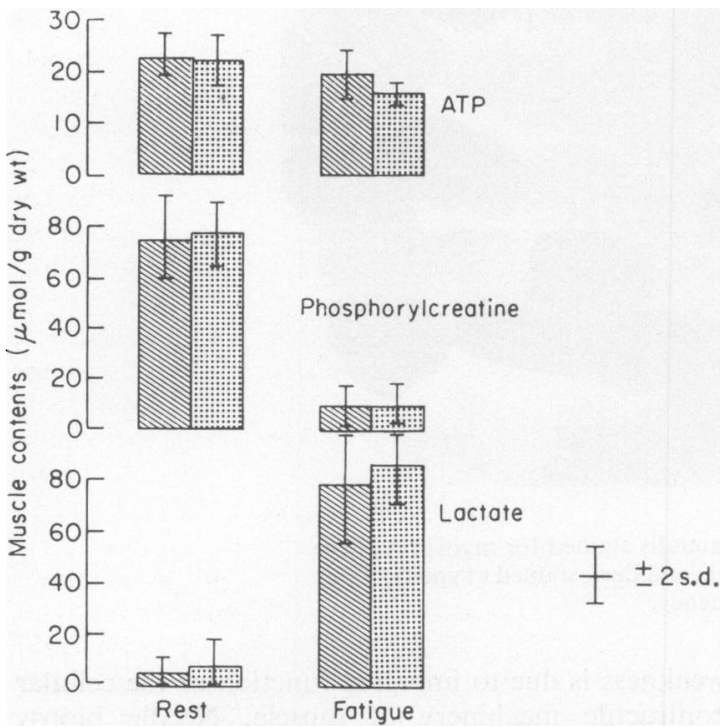

FIG. 5. Analysis of needle biopsy samples of muscle obtained before and at the end of fatiguing isometric contractions of the quadriceps muscle in normal subjects and patients. The patients presented with the complaints of muscle weakness and/or fatigue but EMG and histological, histochemical and electronmicroscopical studies on needle biopsy samples revealed no abnormalities. Note the similar pattern of changes in both groups indicating that the patients had a normal ability to make use of muscle energy supply processes. Normal males (11), hatched; male patients (5), stippled.

The relation between rise in lactate (indicating the contribution of anaerobic glycolysis) and phosphagen (ATP + phosphorylcreatine) depletion, here used as the 'internal' standard of metabolic activity, is shown in Fig. 6. Hyperthyroid patients had values in the normal range. As expected, the patient with McArdle's Disease (McArdle, 1951) which is characterized by a lack of myophosphorylase (Mommaerts et al., 1959) did not produce lactate despite a fall in phosphagen. The patients with hypothyroid myopathy and alcoholic myopathy had reduced muscle lactate production during activity. Evidence of impaired anaerobic glycolysis has already been reported in some alcoholic patients (Perkoff, Hardy and Velez-Garcia, 1966) but not as such in hypothyroid muscle disease.

The finding of a partial impairment of anaerobic glycolysis is interesting but to take this matter further

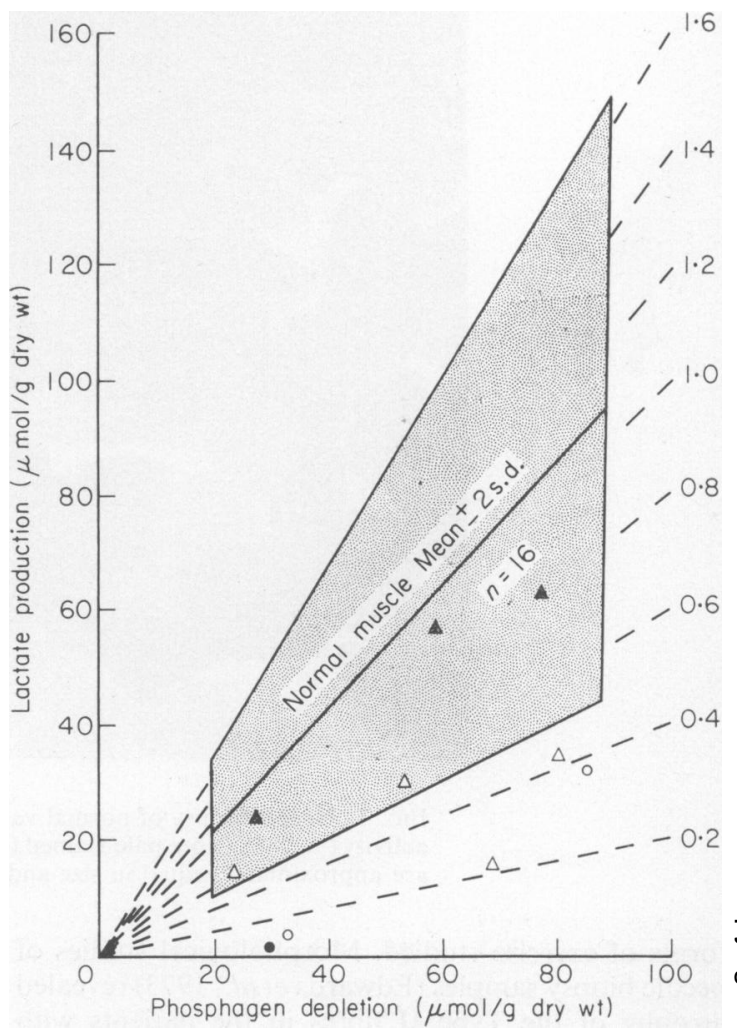

Flg. 6. Relation between muscle lactate production and depletion of phosphagen (ATP + phosphorylcreatine) during fatiguing isometric contractions of quadriceps muscle in patients with metabolic myopathies. Shaded area represents the results for normal muscle (patients and normal subjects) given in Fig. 5. Metabolic myopathies: hyperthyroid, $\mathbf{A}$; hypothyroid, $\Delta$; alcoholic, O; McArdle's disease,

it is necessary to consider muscle structure and how it is altered in disease. Normal muscle comprises two main fibre populations (Fig. 7) when typed according to myosin ATPase activity (Dubowitz and Brooke, 1973). Type I fibres have a low ATPase activity but are rich in myoglobin, mitochondria and oxidative enzymes and are generally slow contracting. Type II fibres have a high ATPase activity, a high glycolytic activity and are generally fast contracting. It appears from recent studies in man that Type I fibres are recruited for long sustained isometric contractions at low forces (Gollnick et al., 1974a) and in dynamic exercise of low intensity (Gollnick, Piehl and Saltin, 1974b). Type II fibres are recruited for higher contraction forces and in dynamic exercise of high intensity. An interesting possibility raised by these investigations is that depletion of energy stores in one or other of the muscle fibre types might be responsible for fatigue in the various 


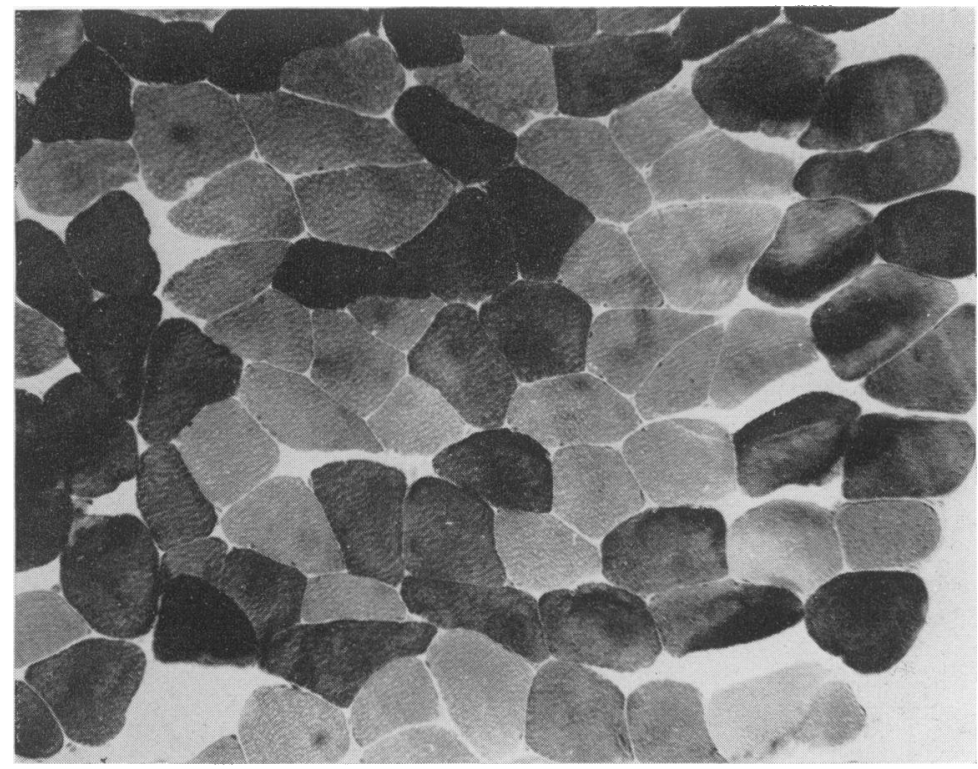

Fig. 7. Needle biopsy of normal vastus lateralis stained for myosin ATPase activity $(\times 288)$. Note pale stained (Type I) and dark stained (Type II) fibres are approximately equal in size and frequency.

forms of exercise studied. Morphological studies of needle biopsy samples (Edwards et al., 1973) revealed atrophy of the Type II fibres in the patients with hypothyroid and alcoholic myopathies. Whether the Type II fibre atrophy, a nonspecific finding in a number of other conditions, simply reflects disuse, with selective preservation of the Type I fibres with a low recruitment threshold is not known with any certainty, though this is clearly a possible explanation. The observed impairment in anaerobic glycolysis in these patients could be due to the reduced volume of Type II (glycolytic) fibres in muscle but this may be too simple an explanation since no information is available for the relative glycolytic rates of the two fibre types in human muscle. A new development which may be helpful is to study the metabolic characteristics of the individual fibre types in needle biopsy samples from patients with metabolic myopathies. Glycogen determinations have already been made in single fibre fragments of normal muscle (Essén and Henriksson, 1974) and preliminary studies of our own suggest that this approach could also be used for the study of other metabolic characteristics of individual fibre types in diseased human muscle.

There are many unsolved problems associated with human muscle fatigue. Quantitation of muscle function in simple clinical tests could help in the evaluation of therapies, while detailed physiological and metabolic studies may reveal the extent to which weakness is due to impaired function of the cellular contractile machinery of muscle. Needle biopsy makes the study of muscle structure and chemistry feasible in a wider range of clinical conditions than has hitherto been possible with open biopsy. It is too early to expect solutions to many of the problems presented by muscle disease but the research tools described are giving promising results which it is hoped will aid in a more fundamental understanding of myopathies and help in the evaluation of possible treatments.

\section{Acknowledgments}

Research described in this paper has been supported by the Wellcome Trust and the Muscular Dystrophy Group of Great Britain.

\section{References}

Ahlborg, B., Bergström, J., Guarnieri, G., Harris, R.C., Hultman, E. \& NordesJö, L.-O. (1972) Muscle metabolism during isometric exercise performed at constant force. Journal of Applied Physiology, 33, 224.

BarcrofT, H. \& Millen, J.L.E. (1939) The bloodflow through muscle during sustained contractions. Journal of Physiology, 97, 17.

BergStröm, J. (1962) Muscle electrolytes in man. Scandinavian Journal of Clinical Investigation, Supplement 68, 11.

DesmedT, J.E. (1973) Editor. New Developments in EMG and Clinical Neurophysiology. Vols 1-3. Karger: Basel.

Dubowitz, V. \& Brooke, M.H. (1973) Muscle Biopsy: A Modern Approach, p. 44. W. B. Saunders: London.

EDWARDS, R.H.T. (1971) Percutaneous needle biopsy of skeletal muscle in diagnosis and research. Lancet, ii, 593. 
EDWARDS, R.H.T. (1973) Film: percutaneous needle biopsy of skeletal muscle in diagnosis and research. Journal of Physiology, 231, 60P.

Edwards, R.H.T., Harris, R.C., Hultman, E., KaiJser, L., KoH, D. \& Nordessö, L.-O. (1972a) Effect of temperature on muscle energy metabolism and endurance during successive isometric contractions, sustained to fatigue, of the quadrice ps muscle in man. Journal of Physiology, 220, 335.

Edwards, R.H.T., Hill, D.K., Jones, D.A. \& Merton, P.A. (1974a) Fatigue of excitation-contraction coupling after prolonged stimulation of human muscle. Abstracts of $X X V I$ International Congress of Physiological Sciences, New Delhi, October, 1974.

Edwards, R.H.T., Hill, D.K. \& McDonnell, M. (1972b) Myothermal and intramuscular pressure measurements during isometric contractions of the human quadriceps muscle. Journal of Physiology, 224, 58P.

Edwards, R.H.T., Maunder, C., Lewis, P.D. \& Pearse, A.G.E. (1973) Percutaneous needle-biopsy in the diagnosis of muscle diseases. Lancet, ii, 1070.

EdWARDS, R.H.T. \& MCDonNell, M. (1974a) A hand-held clinical dynamometer for evaluating voluntary muscle function in patients. Lancet, ii, 757.

Edwards, R.H.T., MCDonNELl, M.J. \& Hill, D.K. (1974b) A thermistor probe for myothermal measurements in man. Journal of Applied Physiology, 36, 4.

Essén, B. \& Henriksson, J. (1974) Glycogen content of individual muscle fibres in man. Acta physiologica scandinavica, 90, 645.

Fessel, W.J., TAYLOR, J.A. \& Johnson, E.S. (1970) Evaluating the complaint of muscle weakness. Simple quantitative clinical tests. In: Muscle Diseases (Ed. by J. N. Walton, N. Canal and G. Scarlato), p. 544. Excerpta Medica: Amsterdam.

Gollnick, P.D., Armstrong, R.B., Saubert IV, C.W., Piehl, K. \& SAltin, B. (1972) Enzyme activity and fiber composition in skeletal muscle of untrained and trained men. Journal of Applied Physiology, 33, 312.
Gollnick, P.D., Karlsson, J., Piehl, K. \& Saltin, B. (1974a) Selective glycogen depletion in skeletal muscle fibres of man following sustained contractions. Journal of Physiology, 241, 59.

Gollnick, P.D., Piehl, K. \& Saltin, B. (1974b) Selective glycogen depletion pattern in human muscle fibres after exercise of varying intensity and at varying pedalling rates. Journal of Physiology, 241, 45.

Hultman, E. (1967) Studies on muscle metabolism of glycogen and active phosphate in man with special reference to exercise and diet. Scandinavian Journal of Clinical Laboratory Investigation, 19, Supplementum, 94.

KARLSSON, J. (1971) Lactate and phosphagen concentrations in working muscle of man. Acta physiologica scandinavica, Supplementum, 358.

MCARde, B. (1951) Myopathy due to a defect in muscle glycogen breakdown. Clinical Science, 10, 13.

Medical Research Council (1943) Aids to the investigation of peripheral nerve injuries, War Memorandum No. 7. Second edition. HMSO: London.

Mommaerts, W.F.H.M., Illingworth, B., Pearson, C.M., Guillory, J. \& Seraydarian, K. (1959) A functional disorder of muscle associated with the absence of phosphorylase. Proceedings of the National Academy of Sciences of the United States of America, 46, 791.

Perkoff, G.T., Hardy, P. \& Velez-Garcia, E. (1966) Reversible acute muscular syndrome in chronic alcoholism. New England Journal of Medicine, 274, 1277.

ROHMERT, W. (1960) Ermittlung von Erholungspausen für statische Arbeit des Menschen. Internationale Zeitschrift für angewandte Physiologie, 18, 123.

Simonson, E. (1971) In: Physiology of Work Capacity and Fatigue. Thomas Springfield: Illinois.

TORNVALL, G. (1963) Assessment of physical capabilities with special reference to the evaluation of maximal voluntary isometric muscle strength and maximal working capacity. Acta physiologica scandinavica, 58, Supplementum, 201. 